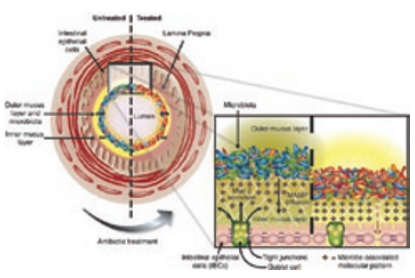

p 98

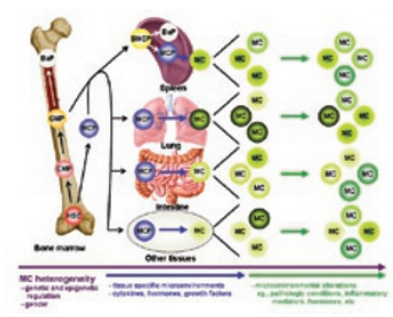

p 111

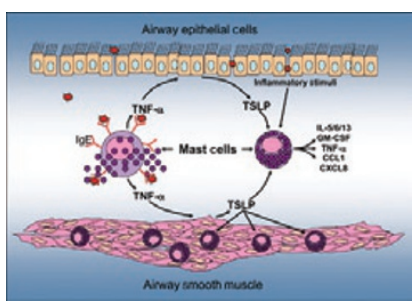

p 138
Intestinal $\gamma \delta$ T cells cells: be specific!

In this month's News \& Highlights, Leo Lefrançois discusses the latest advances and conundrums regarding our understanding of $\gamma \delta$ T cells.

See page 98

\section{Antibiotics and barrier dysfunction}

Marta Wlodarska and B. Brett Finlay discuss how antibiotic-induced changes in the intestinal microbiota may result in disruption of innate host defense mechanisms. See page 100

\section{Activation of innate immunity by allergens}

Marsha Wills-Karp and colleagues discuss the structure and function of allergens, particularly with regard to their fundamental ability to activate the innate immune system by a variety of mechanisms. See page 104

\section{Diverse functions of mast cells}

In this comprehensive review, Tae Chul Moon and colleagues discuss the often-unappreciated roles of mast cells in innate and adaptive immunity and tissue homeostasis. See page 111

\section{Basophils and type 2 immunity}

Caroline Sokol and Ruslan Medzhitov discuss the role of basophils in the induction and regulation of Thelper cell type 2-mediated adaptive immune responses. See page 129

\section{TSLP as a primary regulator of allergic inflammation}

Epithelial cells can affect immune responses through the production of immunoregulatory factors. Mike Comeau and Steve Ziegler discuss the production and function of thymic stromal lymphopoietin (TSLP) in allergic inflammation and its association with human disease. See page 138

\section{Metagenomic analysis of the intestinal microbiota}

David Hill and colleagues employ a metagenomic approach to correlate immune cell homeostasis with changes in bacterial colonization in the gut following antibiotic therapy. See page 148

\section{A novel antigen delivery system for intranasal neonatal vaccination}

Karina Ramirez and co-workers used nonliving, non-genetically modified particles from Lactococcus lactis (called Gram-positive enhancer matrix particles) to intranasally vaccinate neonates and demonstrate lasting protection from infectious challenge. See page 159

\section{Gut T-cell fate in HIV patients}

Emily Ciccone and colleagues demonstrate differences in T-cell numbers and activation status in blood vs. intestinal tissues of HIV patients undergoing antiretroviral therapy. See page 172

\section{Inducing mucosal immunity with cell-based vaccines}

Natasa Strbo and colleagues describe a potent method for mucosal immunization using cell-based gp96-immunoglobulin-secreting vaccines.

See page 182

\section{A systems-biology approach reveals how dendritic cells} operate

Pierluigi Bonifazi and colleagues use a smallinterfering-RNA approach to understand intracellular signaling pathways and dendritic cell-mediated immune regulation during Aspergillus infection in the lung. See page 193 